\title{
Cardiac Regeneration: the Heart of the Issue
}

\author{
Felicia Carotenuto $^{1,2}$ (D) $\cdot$ Vittorio Manzari $^{1} \cdot$ Paolo Di Nardo ${ }^{1,2,3}$
}

Accepted: 9 February 2021 / Published online: 23 March 2021

(C) The Author(s) 2021

\begin{abstract}
Purpose of Review The regenerative capacity of the heart is insufficient to compensate for the pathological loss of cardiomyocytes during a large injury, such as a myocardial infarction. Therapeutic options for patients after cardiac infarction are limited: treatment with drugs that only treat the symptoms or extraordinary measures, such as heart transplantation. Cell therapies offer a promising strategy for cardiac regeneration. In this brief review, the major issues in these areas are discussed, and possible directions for future research are indicated.

Recent Findings Cardiac regeneration can be obtained by at least two strategies: the first is direct to generate an ex vivo functional myocardial tissue that replaces damaged tissue; the second approach aims to stimulate endogenous mechanisms of cardiac repair. However, current cell therapies are still hampered by poor translation into actual clinical applications.

Summary In this scenario, recent advancements in cell biology and biomaterial-based technologies can play a key role to design effective therapeutic approaches.
\end{abstract}

Keywords Cardiac cell therapy $\cdot$ Cardiac regeneration $\cdot$ Biomaterial-based technologies $\cdot 3 \mathrm{D}$ bioprinting

\section{Introduction}

Cardiovascular diseases represent the most important cause of mortality and morbidity in industrialized and developing countries [1]. However, in some of them, the implementation of complex multifaceted medical interventions imbricating aggressive prevention, effective organization of a universal health service, and aggressive application of visionary technologies has induced a progressive containment of the impact of cardiovascular diseases. Indeed, changing people habits through prevention programs is very difficult and requires

This article is part of the Topical Collection on Cellular Transplants

Felicia Carotenuto

carotenuto@med.uniroma2.it

Paolo Di Nardo

dinardo@uniroma2.it

Vittorio Manzari

manzari@uniroma2.it

1 Dipartimento di Scienze Cliniche e Medicina Traslazionale, Università di Roma "Tor Vergata", Rome, Italy

2 Centro Interdipartimentale per la Medicina Rigenerativa (CIMER), Università di Roma "Tor Vergata", Rome, Italy

3 L.L. Levshin Institute of Cluster Oncology, I.M. Sechenov First Moscow State Medical University, Moscow, Russia the aptitude to modify the society at the personal and collective level. Instead, the organization of an efficient health service able to protect on equal bases all citizens is the responsibility of governments that must allocate considerable human and financial resources in this direction. Finally, the massive incorporation of technology after the II World War has determined a dramatic improvement in diagnostics and therapies causing a substantial decline in cardiovascular mortality, but not in the disease prevalence.

The reduced death rate has mostly been determined by the administration of sophisticated long-term palliative treatments (drugs, pacemakers, stents, etc.), being heart transplantation the only radical treatment, regrettably reserved to few patients worldwide. On the other hand, myocardial self-overhauling capability is very limited, so the natural fate on an injured myocardium is the substitution of the contractile cells with fibrosis that induces a progressive decline in cardiac function and the consequent heart failure.

In this context, more than 25 years ago [2•], it has been envisioned the possibility to circumvent this deadlock by administering living cells, or some of their components, to the injured tissue to reconstitute, at least functionally, its integrity. The implementation of a cell-based therapy could make available very efficient treatments at reduced costs to all cardiovascular patients avoiding current limits determined by donor 
shortage. Indeed, many progresses have been achieved in this direction, but, at present, no treatment efficient in preserving heart function has been brought to the bedside in spite of years of intensive investigative efforts and huge worldwide investments. Maybe, a simplistic approach based on initial inconsistent hopes has represented the major factor hampering the clinical exploitation, while the innate complexity of the cardiac cell therapy deserves an extraordinary attention to all details of the whole procedure [3•].

Regenerative strategies have been evolutionarily elaborated by all living systems to preserve the structure and function of the different tissues and organs. However, the prowess to repair/regenerate is not the same in the different species, and in the diverse organs and tissues. The rate of cardiac regeneration is high in teleost fish, moderate in urodele amphibians, and almost negligible in mammals [4]. Among others, in mammals, such as mice and humans [5], within a few days after birth, the myocardium displays a remarkable potential to repair/regenerate, while, in adult mammals, the cardiomyocyte turn-over is very low in physiological conditions and not able to meet in a meaningful time interval the massive cell demand (1-10 billion dead cells) that suddenly arises after an ischemic attack. As a consequence, the myocardium compensates for the loss of contractile cells with an extensive fibrosis that alters the geometry of heart cavities and their capability to propel the blood, ultimately determining the heart failure. To counteract the progression of the above-mentioned disease, sophisticated treatments have been optimized over the last decades with the aim of restoring the blood supply to the myocardium, while reducing the fibrosis and, thus, preserving heart function. However, these treatments are palliative, since they do not cure the diseases, but slow down the progression of the illness expanding longevity in spite of the marked increase in prevalence $[6,7]$. When their effects are no more able to delay the negative consequences of the disease, few patients only can be treated with heart transplantation owing to donor shortage, possible rejection, and very high costs of surgery. This means that millions of patients suffering from life-threatening heart failure cannot be cured every year.

The outstanding advancements in stem cell biology in early 2000s suggested the idea that, after implantation, adult stem cells could "transdifferentiate" into the cells of a host tissue driven by the recipient new microenvironment. Divisive results were generated to confirm the possibility that bone marrow hematopoietic stem cells could transdifferentiate into cardiomyocytes repairing the injured myocardium in preclinical models [8, 9], while uncontrolled clinical trials showed little, if any, beneficial effects on cardiac function [10••, 11••]. Also, studies and clinical trials carried on using stem cells isolated from the myocardium failed to show significant engraftment and integration in the injured hosting tissue [12•]. However, a meager improvement in cardiac function was observed in these studies and has been later attributed to the release of bioactive factors entrapped into extracellular vesicles or in solution into the ECM that can spur the cardiac function [13-15•].

Nevertheless, after 20 years of intensive studies heavily funded by public and private organizations, no clinically reliable protocols for cardiac regeneration have been generated by the innumerable laboratories involved, all subjugated by the fake and pernicious discoveries trumpeted by a preeminent tiny group of scientists supported by major scientific journals and research institutions [16]. As result, an inexorable lack of credibility and enthusiasm about the potential of cell therapy to cure heart failure has spread through research centers worldwide with prejudice in respect to this visionary research field. Indeed, a dispassionate analysis of what has happened demonstrates that, although a simplistic and unsystematic approach has been applied to study very complex subjects submissively conforming to unsubstantiated assumptions, a reliable answer to the question of whether the tissue regenerative potential could be exploited to treat severe heart diseases is still missing. Thus, it could be worth trying again applying the most rigorous scientific principles. In this context, the major issues to be addressed before the cardiac cell therapy could be currently used at the bedside are discussed, and possible directions for future research are indicated in the following.

\section{Cells as Therapeutic Agents}

Several cell types from intra- and extra-myocardial sources have been indicated as potentially able to regenerate the myocardium. These cell types include (i) adult stem cells (ASCs) [i.e., skeletal myoblasts, bone marrow-derived mononuclear cells (BMMNCs), mesenchymal stem (or stromal) cells (MSCs), hematopoietic stem cells (HSCs), endothelial progenitor cells (EPCs), cardiac progenitor cells (CPCs), epicardium-derived cells, cardiac side population cells, stem cell antigen-1 - Sca-1 + CPCs, pericytes, adipose stem cells] and (ii) pluripotent stem cells (PSCs) [i.e., embryonic stem cells (ESCs) and induced pluripotent stem cells (iPS)]. The iPS cells share the same characteristic of ESCs, but are generated from patient-specific somatic cells, bypassing ethical concerns associated with the use of ESCs and providing an autologous source of human cells [17••].

During the past 2 decades, several clinical trials, mainly testing ASCs, have failed to show new contractile myocardium and reproducible improvement in cardiac function. The prevailing consensus now is that some beneficial effects are mediated through paracrine factors secreted by the implanted cells, such as cytokines, and growth factors that stimulate new blood vessel formation and reduce postinfarct inflammation. Indeed, all the ASCs implanted into the myocardium have demonstrated only a limited capability to generate cardiomyocytes $[11 \bullet \bullet$, but it is not clear whether this inability 
is because the real progenitor is missed or technical issues, during their isolation and expansion, have suppressed cell progenitorial characteristics. In fact, independently of the source, the isolation protocol is based on the extraction of putative stem cells from their special native tissue microenvironment. Once progenitor cells are isolated and expanded in two-dimensional cultures in vitro, they lose most of the interactions occurring in the three-dimensional microenvironment, which are fundamental in the control of cell fate decisions. Afterwards, isolated putative stem cells are identified by the expression of different molecular markers, none of which is rigorously cell-specific. De facto, the "stemness" is evaluated in vitro through functional assay (i.e., the capability to differentiate into osteoblasts, adipocytes, and cardiomyocytes). In addition, cells maintained in long-term culture in standard conditions (i.e., bi-dimensional flasks and culture medium supplemented with serum) develop mild immunogenicity, also in autologous cells, altered differentiation behavior and epigenetic modifications $[18,19]$ as well as gap junction alterations [20]. This condition is further stressed by the absence of the extracellular matrix (ECM) that supplies cells with key physical and biochemical signals regulating cell behavior.

The ECM is a fiber-reinforced hydrogel composite of structural and functional proteins, polysaccharides, and multiadhesive glycoproteins produced and deposited by resident cells, whose weak interactions guide gel self-assembly and integrity. ECM composition depends on tissue type and determines its mechanical and physiological role. Cells and ECM are reciprocally and dynamically connected by a multitude of mechanophysical and biomolecular signals that go from outside to inside the cell and vice versa. Thus, cells continually remodel the ECM present in their microenvironment, and these dynamic modifications of the ECM direct cell fate [21]. In this context, after isolation, stem cells are deprived by their unique ECM with consequent derating of their prowess to differentiate and integrate into the hosting tissue. So, until adequate protocols able to maintain optimal environmental conditions outside the original tissue are setup, the actual potency and behavior of putative cardiac progenitor cells remain uncertain. The definition of this crucial point implies that novel reliable markers defining an irrefutable fingerprint of "stemness" must be identified, so that also the purity of the isolated population is guaranteed. In addition, novel culture procedures based on spatio-temporal algorithms to regulate the release of biological and physical signals to stem cells must be invented to adequately expand (until 1-10 billion cells/treatment) and address cell differentiation and orientation. As result, a pure and genetically stable cardiomyocyte population must be generated that couples mechanically and electrically to the recipient myocardium in order to form a safe long-term functional tissue.

As an alternative to adult cells, human pluripotent stem cells (PSCs) have displayed a great potential to generate differentiated cardiomyocyte which show evidence of direct engraftment and electromechanical coupling with the host myocardium and to functionally remuscularize the infarcted heart of preclinical models including macaque monkeys [22•, 23•]. For this reason, PSC-cardiomyocytes have now emerged as a leading candidate for cardiac regeneration. However, more must be understood to avoid arrhythmias. Electrophysiologic studies suggest the tendency for cell grafts to act as a nidus for arrhythmia. The potential arrhythmic risk may be attributed to the presence of immature cells in the pool of transplanted cells. Indeed, immature or undifferentiated cells exhibit inadequate electrophysiological properties, such as less organized gap junctions that may determine nonfunctional electrical coupling with resident cardiomyocytes [24]. Furthermore, the possible presence of undifferentiated cells raises concern about the risk of tumorigenicity [25]. To overcome these issues, several strategies have been proposed to implement differentiation protocols to generate a pure population of mature cardiomyocytes [26 $6^{\circ}$. However, such processes would be associated with high production costs. Furthermore, despite considerable progress in our knowledge of PSC biology, it is unclear what could be the appropriate maturation cocktail to generate PSC-derived cardiomyocytes with adult-like phenotypes in vitro for transplantation [27•]. These problems hinder the clinical translation of PSC-derived cardiomyocytes.

In the last years, other cell-free strategies based on the regenerating effects of soluble factors and extracellular vesicles such as exosomes released by the injected stem cells [28] while engrafted into the hosting myocardium have been proposed. Consequently, an alternative line of research is based on the concept that the exclusive administration of cell secretome can harness cardiac endogenous repair pathways leading to both stimulation of angiogenesis and mitigation of inflammation and fibrosis [26]. Factors of cell secretome could also be incorporated into biomaterial-based microparticles that allow their prolonged release in order to extend the exposure time of the cardiac tissue [29, 30] (Fig. 1). However, there is still limited knowledge of the ideal mixture of bioactive factors to be locally delivered for restoring heart function, and this promising field of research needs a very careful investigation before any possible clinical application.

\section{Facilitating Cell Engraftment: Immunomodulation of the Post-ischemic Myocardial Environment}

A first crucial factor determining a successful cell engraftment is the post-ischemic myocardial microenvironment. Most of the studies about heart regeneration have been focused on cell source and number and their capability to engraft into the myocardium neglecting the quality of the recipient tissue. After an 
Fig. 1 Cardiac cell therapy strategies. The therapeutic benefits of cell therapy can be mediated both by different bioproducts released by the cells (e.g., exosomes) and by an ex vivo functional myocardial tissue that replaces the damaged tissue. Emerging technologies based on biomaterials can dramatically improve both strategies

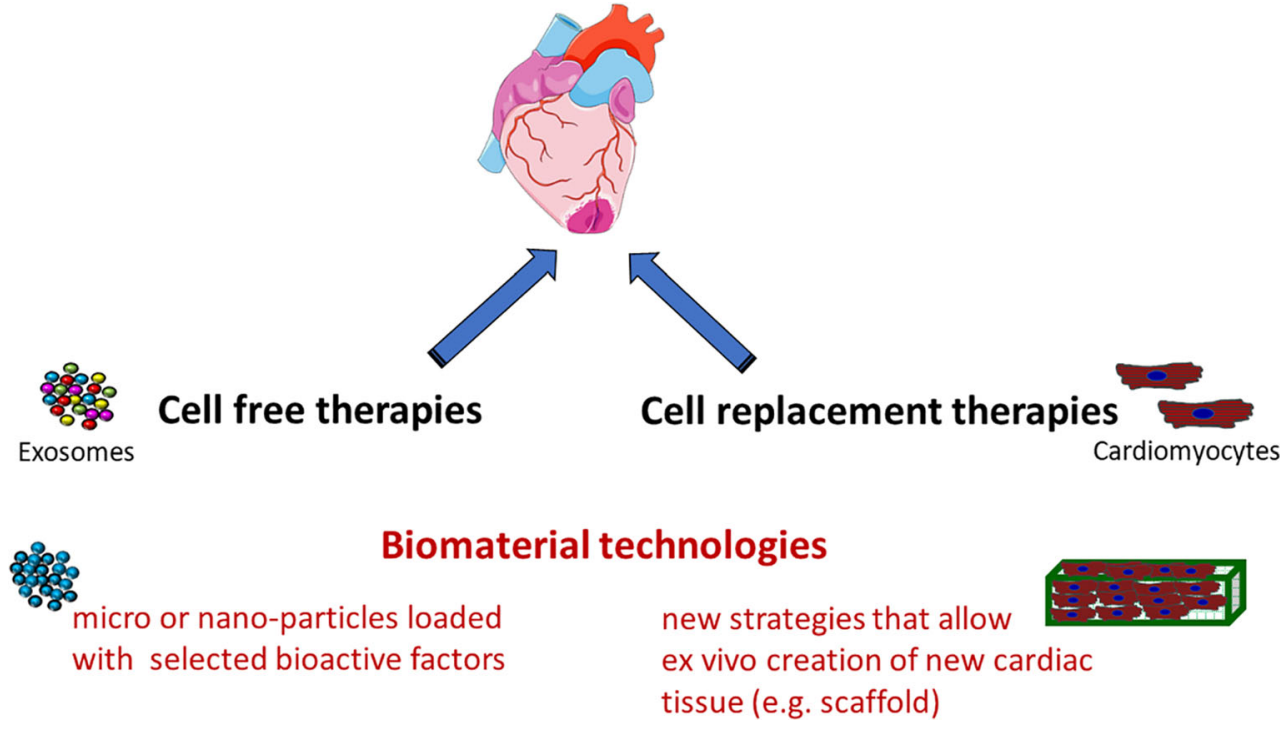

ischemic attack, the myocardium undergoes a multistep adaptation ultimately resulting in the substitution of the contractile tissue with fibrosis, being the regenerative mechanisms unable to repair the heart. The in vivo structural and functional environmental changes caused by the altered interactions at different levels into the post-ischemic myocardium are fatal to the implanted alien cells. The sudden deprivation of the blood supply deprives the myocardium of oxygen, metabolites, and other factors necessary for cell machinery. As a result, $\mathrm{pH}$ and intracellular cascades are undermined, and necrotic and apoptotic processes are triggered. At the same time, "danger signals" by necrotic cardiomyocytes and fragmented ECM activate innate immune pathways and trigger an intense inflammatory response [31]. An intricate collection of soluble factors is activated, and both blood-derived and tissue-resident immune cells invade the damaged tissue. In this context, monocytes are recruited and transdifferentiated into macrophages necessary to remove the cell debris (M1 subpopulation), but also to prompt cardiac angiogenesis, healing, and repair (subpopulation M2) $[32,33]$. In fact, M1 macrophages release pro-inflammatory cytokines, such as TNF and interleukin 6 (IL-6), that are detrimental to surviving cardiomyocytes and evoke extensive remodeling of cardiac tissue through secretion of matrix proteases and activation of myofibroblasts that produce excessive extracellular matrix. Instead, M2 macrophages exhibit an antiinflammatory, pro-regenerative phenotype due to their ability to secrete high levels of anti-inflammatory cytokines including IL-10 and growth factors, such as VEGF [34]. However, if the inflammatory response is excessive, the chronic activation of immune cells may promote fibrosis and hypertrophy. As a consequence, the fibrosis modifies the tissue stiffness, while cells and vascular beds are misaligned, and bioarchitecture is devastated. This unfavorable microenvironment of acute myocardial infarction has a negative effect on the viability of grafted cells [35].
Among the cells which can be transplanted, MSC can escape immune system detection, due to the absence of expression in the MHC class II molecules, while depressing the inflammatory process and enhancing the reparative progression [36]. MSC plays a role in the modulation of inflammation and immune response after myocardial infarction through the release of exosomes, influencing myocardial repair and remodeling [37]. MSC-derived exosomes may reduce cardiac inflammation after myocardial injury modifying the polarization of M1 macrophages to M2 macrophages via miR-182 shuttling [38], decreasing neutrophil infiltration and T-cell proliferation [39]. Similar effects can be induced by CPCderived exosomes reducing leucocyte infiltration [40], while stimulating macrophage polarization towards a M2 antiinflammatory phenotype after myocardial injury [41].

Taken together, it is possible to envision a more efficient strategy based on the modulation of the inflammation of the inhospitable post-ischemic myocardial environment to create suitable conditions in which optimally cultured implanted cells can more easily engraft, differentiate, and integrate with the recipient tissue. This requires that novel pharmacological strategies are implemented to be associated with the administration of MSC- or CPC-derived exosomes and previously in vitro differentiated cells towards a cardiomyocytic phenotype.

\section{Cell Delivery and the Development of Biomaterial-Based Technologies}

Cells have been administered to the injured myocardium as (i) embedded in natural tissue fragments, (ii) harvested from native tissue and then expanded ex vivo before being delivered, and (iii) embedded in engineered tissues manufactured outside the body and then implanted. In most of experiments and 
trials, isolated and conventionally cultured stem cells have been injected into and/or around the area of the myocardial damage. This technique is very cost-effective: the syringe and the procedure have a very low cost and do not require complex skills or training. Unfortunately, only a negligible number of injected cells integrate into the damaged myocardium, with meager, if any, improvement of the contractile function $[11 \bullet, 42]$. Among many potential reasons, it must be considered that cultured cells, deprived of their ECM and floating in the culture medium or physiological solution, are remarkably different in respect to their native siblings. Their behavior is further affected by the traumatic passage through a very narrow needle gauge and the abnormal pressure differential between the syringe inner volume and the intramuscular cardiac environment. Therefore, the injection per se could represent a negative factor contributing to the reduced engrafting potential of progenitor cells. Indeed, initial hypotheses that progenitor cells could be led to a cardiomyocyte phenotype from environmental signals after myocardial transplantation was gradually abandoned and the field shifted instead toward replacing damaged myocardium area with cardiac-committed cells or mature cardiomyocytes.

Cells can be cultured and induced to differentiate on threedimensional polymeric structures (scaffolds) to manufacture patches of tissue outside the body to be implanted in the damaged myocardium. The scaffold technology has rapidly evolved over the years trying to mimic as much as possible the ECM structure and function: different natural, synthetic, and composite materials are scrutinized to manufacture scaffolds able to address cell fate and tissue bioarchitecture [43•].

In particular, a lot of attention is currently paid to design 3D hydrogels able to deliver to cells' physical signals, which are released through appropriate stiffness and texture (pores that allow the oxygen and nutrients flow, and the waste removal), and biological signals conveyed by solute morphogens, growth factors and microvesicles, loaded with proteins and nucleic acid sequences. An example of a very innovative design is the "scaffold-in-scaffold" approach in which a stiffer woodpile structure manufactured using 3D additive technology is embedded into a hydrogel to dynamically release mechano-structural signals to the cells incorporated into the structure in order to modulate cell differentiation and orientation. This 3D poly-(ethylene glycol) diacrylate (PEGDA)based scaffold obtained by micro-stereolithography promoted human cardiac progenitor cell 3D spatial orientation and activation of the expression of $\alpha$-sarcomeric actinin and connexin 43 [44•]. As a further example, a cardiopatch manufactured using porous elastomeric polycaprolactone (PCL) 3D membrane filled with self-assembling peptide hydrogel and seeded with autologous adipose tissue-derived progenitor cells improves myocardial infarct scars in sheep [45].

However, better results are expected using decellularized ECM (dECM) that can be repopulated using iPS-derived cardiac cells [46]. The dECM (i.e., the non-cellular component of tissue) largely retains relevant biological and structural cues that are responsible for optimal cell adhesion, orientation, and differentiation. These dECM-based constructs repopulated by cells promise to generate effective human therapeutic grafts $[47,48]$. However, there is limited capacity to tune physicochemical properties in IECM used as solid scaffolds that maintain native matrix structure. For this reason, more recently, the dECMs have been further processed to generate dECM products as starting materials (e.g., powder and solubilized derivatives, hydrogels, or bioinks) for 3D printing [49-51] or electrospinning processes [52, 53]. These approaches allow for modulation of architecture and mechanical properties of dECM-based scaffolds, leveraging the biochemical cues of native ECM with the capacity to tune physicochemical properties.

Bioactivity of dECM-based scaffolds may be further augmented using strategies for conjugation of bioactive molecules based on click chemistry because "click"reactions" offer high selectivity, versatility, simplicity, and yield [52]. Conductive materials may be incorporated into the scaffolds to improve the myocardium electrophysiological activity and contraction [54]. Finally, next generations of "smart" biomaterials that have the ability to change physiological parameters and exogenous stimuli as a function of time can be employed to achieve hybrid composite constructs with dynamic temporal control [55]. Despite the development of new biomaterials and scaffolds which led to the fabrication of better biomimetic tissues there is still a difficulty in generating large, multicellular, and vascularized tissues with the architectural complexity of native cardiac tissues. In this respect, recent advances in biofabrication technologies, including three-dimensional (3D) bioprinting, have allowed the generation of more complex biological structures at high spatial resolution with integrated vasculature and multiple cells.

\section{D Bioprinting Technologies: Towards the Building of a Whole Heart}

The myocardium is a complex tissue in which cardiomyocytes are interlaced with nonmyocyte cells to generate an intricately organized 3D structure with an elaborate vasculature system and specific physiological, biomechanical, and electrical properties. Currently, the most advanced technique for fabricating myocardial implants endowed with complex biomimetic features recapitulating the tissue native physiochemical and biomechanical characteristics is 3D bioprinting [56]. This is a rapid prototyping and additive manufacturing technique that enables the fabrication with high precision through a layer-bylayer building process of tissue-like constructs replicating the complex architecture of biological systems [57]. Traditional $3 \mathrm{D}$ printing uses metals, plastics, and polymers as printing 
materials or "ink"; instead, bioprinting uses living cells and biological matrix or "bioink" and manufacture is governed by ad hoc "computer-aided design" (CAD) files that provide the blueprint to fabricate the desired tissue construct [58]. Bioprinting technology also allows the fabrication of anatomical shaped 3D structures by using patients' images obtained from medical imaging technologies, such as computer tomography (CT) and magnetic resonance imaging (MRI) [59] (Fig. 2).

Since the debut of the first inkjet bioprinter in 2003 [60••], the bioprinting technology has undergone a rapid evolution. Extrusion-based bioprinting, also known as direct ink writing, which derived from inkjet printing, is the most widely used approach of $3 \mathrm{D}$ bioprinting because of its versatility [61].

Sacrificial bioprinting has been developed to produce tissue blocks encapsulating interconnected hollow channels simulating the vascular network [62]. The development of methods such as "freeform reversible embedding of suspended hydrogels" (FRESH) has enabled for soft biomaterial printing, within a thermoreversible hydrogel support bath, by preventing them from collapsing during the bioink deposition process due to gravity $[63 \bullet \bullet$. Bioprinters equipped with multiple nozzles extruding different biomaterials boosted the capacity to build complex tissues.

A functional cardiac tissue can be obtained via a microfluidic-based printing head (MPH) that allows to precisely tailor cells' 3D spatial deposition, guaranteeing a high printing fidelity and resolution. This multicellular construct which is composed of iPS-CMs and endothelial cells (HUVECs) encapsulated within hydrogel strands containing alginate and PEG-fibrinogen showed vasculature development in vitro and in vivo models [64]. The proof of concept of printing cardiac constructs of clinically relevant size and a whole heart was provided by the recent work of Noor et al. [65••]. A thick $(\approx 2 \mathrm{~mm})$ and vascularized human cardiac tissue was fabricated with cells and ECM material derived entirely from the patient's biopsy (fatty tissue) and therefore with a minimum risk of immune rejection. Cells were reprogrammed into iPS cells and differentiated to cardiomyocytes and endothelial cells. ECM material was processed to form a hydrogel and combined with cells to form a bioink. These vascularized cardiac patches were printed using an extrusion-based bioprinter and fully matched the anatomical and immunological properties of the patient. The cardiac tissue structure
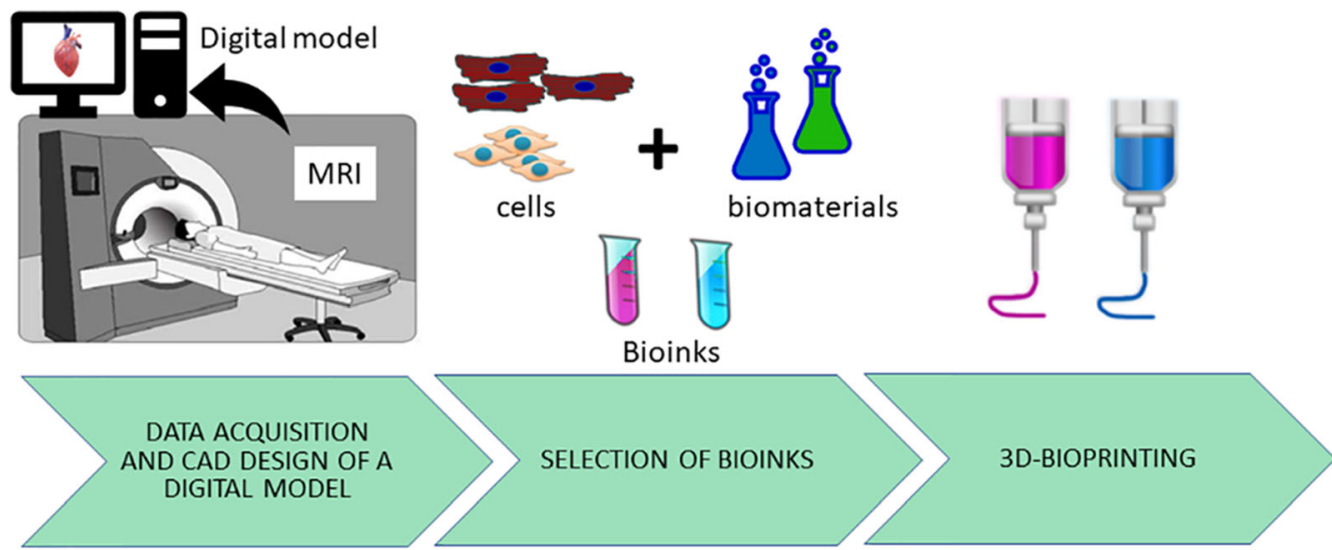

\section{CHALLENGES}

Implemention of cell differentiation protocols

$>$ Generation of billion cells

Refinement of advanced cell culture techniques

Development of new biomaterials

$>$ Improvement of 3D bioprinting technologies to generate complex biological structures with integrated vasculature and multiple cell types at high spatial resolution

Bioreactors for maintenance of bio-printed heart before transplantation

Fig. 2 Schematic representation of 3D bioprinting process. Data acquisition can be obtained by computed tomography or magnetic resonance imaging (MRI). Patient MRI images are used to model the heart by a CAD software. Biomaterials and cells are selected and processed to form bioinks and applied to bioprint patient-specific human hearts. Bioprinted hearts are conditioned in bioreactors, functionalized, and used for transplantation 
was designed by a CAD software that used the patient's anatomical data from the images obtained by a computed tomography. Furthermore, a cellularized human heart with a natural architecture of the size of a rabbit heart (height $20 \mathrm{~mm}$, diameter $14 \mathrm{~mm}$ ) was printed which was perfusable, but functionally unable to pump blood. Indeed, whole-heart bioprinting lacked the placement of smaller structures, such as microvascularization and nerves.

Nevertheless, bioprinting of collagen components of human hearts at various scales (from capillaries to the full organ) based on optimized FRESH technology was recently achieved by Lee et al. [66••]. Cardiac ventricles printed with human cardiomyocytes accurately reproduce patient-specific anatomical structure and showed synchronized contractions. FRESH bioprinting was also used to produce a patient-specific anatomical model of an adult human heart at full size using tunable alginate bioinks $[67 \bullet \bullet]$. Though this printed heart mimicked the mechanical properties of cardiac tissue, it was not cellularized and therefore only useful as a surgical training tool. Indeed, billions of cells would be required to 3Dbioprint whole organs.

These achievements demonstrate that bioprinting techniques have evolved significantly paving the way for the printing of complex organs, such as the heart, but more remains to be done to fabricate fully functional organs for transplantation.

\section{Conclusions}

Disproportionate hopes have been propagated so far about the possibility to adopt cell therapy to cure severe heart failure in an egotistic vision unsubstantiated by robust scientific fundaments. The superior complexity of this research domain, instead, requires great passion and ingenuity in a long-term effort characterized by a humble, progressive, and constant approach. In this context, a key need to determine a paradigm shift is the transdisciplinarity that can provide specific solutions to the major challenges of the multiple dominant mechanisms regulating cell fate and its exploitation in the clinical setting. However, encouraging preliminary results indicate that further advances in cell biology, tissue engineering, biomaterial technologies, and digitalized fabrication techniques (3D bioprinting) could finally allow the fabrication of biological tissues with structural and compositional accuracy adequate for clinical applications.

Funding Open access funding provided by Università degli Studi di Roma Tor Vergata within the CRUI-CARE Agreement. This work was supported by "Mission: Sustainability" Grant (DR 2817/2016), Progetto:
Biomechanical scaling stimula: inducing differentiation (BIOMESSID), "Tor Vergata" University of Rome (Italy).

\section{Declarations}

Conflict of Interest All authors declare no competing interests.

Open Access This article is licensed under a Creative Commons Attribution 4.0 International License, which permits use, sharing, adaptation, distribution and reproduction in any medium or format, as long as you give appropriate credit to the original author(s) and the source, provide a link to the Creative Commons licence, and indicate if changes were made. The images or other third party material in this article are included in the article's Creative Commons licence, unless indicated otherwise in a credit line to the material. If material is not included in the article's Creative Commons licence and your intended use is not permitted by statutory regulation or exceeds the permitted use, you will need to obtain permission directly from the copyright holder. To view a copy of this licence, visit http://creativecommons.org/licenses/by/4.0/.

\section{References}

Papers of particular interest, published recently, have been highlighted as:

- Of importance

•- Of major importance

1. Benjamin EJ, Muntner P, Alonso A, Bittencourt MS, Callaway CW, Carson AP, et al. Heart disease and stroke statistics-2019 update: a report from the American Heart Association. Circulation. 2019;139(10):e56-e528.

2. Claycomb WC, Di Nardo P. Cardiac growth and regeneration. In: Annals of the New York Academy of Sciences, vol. 752. (ITALY): Rome Univ; 1996. Book based on a workshop on cardiac growth and regeneration, held in Viterbo, Italy on June 1994. The first pioneering studies are described.

3. Carotenuto F, Teodori L, Maccari AM, Delbono L, Orlando G, Di Nardo P. Turning regenerative technologies into treatment to repair myocardial injuries. J Cell Mol Med. 2020;24(5):2704-16. Thorough overview of current barriers, limitations and potential solutions of the cell cardiac therapies.

4. Ausoni S, Sartore S. From fish to amphibians to mammals: in search of novel strategies to optimize cardiac regeneration. J Cell Biol. 2009;184(3):357-64.

5. Haubner BJ, Schneider J, Schweigmann U, Schuetz T, Dichtl W, Velik-Salchner C, et al. Functional recovery of a human neonatal heart after severe myocardial infarction. Circ Res. 2016;118(2): 216-21.

6. Machaj F, Dembowska E, Rosik J, Szostak B, Mazurek-Mochol M, Pawlik A. New therapies for the treatment of heart failure: a summary of recent accomplishments. Ther Clin Risk Manag. 2019;15: 147-55.

7. Menasche P. Cell therapy trials for heart regeneration - lessons learned and future directions. Nat Rev Cardiol. 2018;15(11):659 71.

8. Orlic D, Kajstura J, Chimenti S, Jakoniuk I, Anderson SM, Li B, et al. Bone marrow cells regenerate infarcted myocardium. Nature. 2001;410(6829):701-5.

9. Murry CE, Soonpaa MH, Reinecke H, Nakajima H, Nakajima HO, Rubart M, et al. Haematopoietic stem cells do not transdifferentiate 
into cardiac myocytes in myocardial infarcts. Nature. 2004;428(6983):664-8.

10.• A futile cycle in cell therapy. Nat Biotechnol. 2017;35(4):291. In this editorial, the editors expressed a severe concern on the benefits of cardiac cell therapy trials.

11.• Eschenhagen T, Bolli R, Braun T, Field LJ, Fleischmann BK, Frisen J, et al. Cardiomyocyte regeneration: a consensus statement. Circulation. 2017;136(7):680-6. Important publication in which the authors try to define areas of agreement, and areas requiring further elucidation related to the regenerative potential of the myocardium.

12. van Berlo JH, Kanisicak O, Maillet M, Vagnozzi RJ, Karch J, Lin $\mathrm{SC}$, et al. c-kit+ cells minimally contribute cardiomyocytes to the heart. Nature. 2014;509(7500):337-41.

13. Gnecchi M, Zhang Z, Ni A, Dzau VJ. Paracrine mechanisms in adult stem cell signaling and therapy. Circ Res. 2008;103(11): 1204-19. Key experiments that observed the beneficial effects induced by stem cell- conditioned media on cardiomyocytes supporting 'paracrine hypothesis' in cardiac cell therapy.

14. Takahashi M, Li TS, Suzuki R, Kobayashi T, Ito H, Ikeda Y, et al. Cytokines produced by bone marrow cells can contribute to functional improvement of the infarcted heart by protecting cardiomyocytes from ischemic injury. Am J Physiol Heart Circ Physiol. 2006;291(2):H886-93. Key experiments that observed the beneficial effects induced by stem cell-conditioned media on the infarcted myocardium supporting the 'paracrine hypothesis' in cardiac cell therapy.

15. Hodgkinson CP, Bareja A, Gomez JA, Dzau VJ. Emerging concepts in paracrine mechanisms in regenerative cardiovascular medicine and biology. Circ Res. 2016;118(1):95-107. Overview of mechanisms through which paracrine factors released by stem cells promote cardiac repair and regeneration.

16. Bolli R, Tang XL, Guo Y, Li Q. After the storm: an objective appraisal of the efficacy of c-kit+ cardiac progenitor cells in preclinical models of heart disease. Can J Physiol Pharmacol. 2020:111.

17.• Takahashi K, Yamanaka S. Induction of pluripotent stem cells from mouse embryonic and adult fibroblast cultures by defined factors. Cell. 2006;126(4):663-76. Milestone of scientific literature on regenerative medicine that describes the first reported generation of mouse induced pluripotent stem cells.

18. Zhao T, Zhang ZN, Rong Z, Xu Y. Immunogenicity of induced pluripotent stem cells. Nature. 2011;474(7350):212-5.

19. Lister R, Pelizzola M, Kida YS, Hawkins RD, Nery JR, Hon G, et al. Hotspots of aberrant epigenomic reprogramming in human induced pluripotent stem cells. Nature. 2011;471(7336):68-73.

20. Almeida SO, Skelton RJ, Adigopula S, Ardehali R. Arrhythmia in stem cell transplantation. Card Electrophysiol Clin. 2015;7(2):35770 .

21. Nicolas J, Magli S, Rabbachin L, Sampaolesi S, Nicotra F, Russo L. 3D Extracellular matrix mimics: fundamental concepts and role of materials chemistry to influence stem cell fate. Biomacromolecules. 2020;21(6):1968-94.

22. Weinberger F, Breckwoldt K, Pecha S, Kelly A, Geertz B, Starbatty J, et al. Cardiac repair in guinea pigs with human engineered heart tissue from induced pluripotent stem cells. Sci Transl Med. 2016;8(363):363ra148. This study displays the great ability of differentiated cardiomyocyte generated from human iPS to functionally remuscularize infarct tissue in guinea pigs.

23. Liu YW, Chen B, Yang X, Fugate JA, Kalucki FA, FutakuchiTsuchida A, et al. Human embryonic stem cell-derived cardiomyocytes restore function in infarcted hearts of non-human primates. Nat Biotechnol. 2018;36(7):597-605. This paper demonstrates the ability of human embryonic stem cell-derived cardiomyocytes to functionally remuscularize infarct tissue in macaque monkeys.
24. Chen K, Huang Y, Singh R, Wang ZZ. Arrhythmogenic risks of stem cell replacement therapy for cardiovascular diseases. J Cell Physiol. 2020;235(9):6257-67.

25. Sato Y, Bando H, Di Piazza M, Gowing G, Herberts C, Jackman S, et al. Tumorigenicity assessment of cell therapy products: the need for global consensus and points to consider. Cytotherapy. 2019;21(11):1095-111.

26. $\mathrm{Tu}$ C, Chao BS, Wu JC. Strategies for improving the maturity of human induced pluripotent stem cell-derived cardiomyocytes. Circ Res. 2018;123(5):512-4. This editorial underline the need to develop strategies, including both physical and biochemical stimulations, to generate a pure population of mature cardiomyocytes from PSC.

27. Karbassi E, Fenix A, Marchiano S, Muraoka N, Nakamura K, Yang $\mathrm{X}$, et al. Cardiomyocyte maturation: advances in knowledge and implications for regenerative medicine. Nat Rev Cardiol. 2020;17(6):341-59. This recent overview shows and discuss the current different approaches to mature PSC-derived cardiomyocytes.

28. Gebara N, Rossi A, Skovronova R, Aziz JM, Asthana A, Bussolati B. Extracellular vesicles, apoptotic bodies and mitochondria: stem cell bioproducts for organ regeneration. Curr Transplant Rep. 2020;7(2):105-13.

29. Caso MF, Carotenuto F, Di Nardo P, Migliore A, Aguilera A, Lopez CM, et al. Nanoporous microsponge particles (NMP) of polysaccharides as universal carriers for biomolecules delivery. Nanomaterials (Basel). 2020;10(6).

30. Corsi F, Carotenuto F, Di Nardo P, Teodori L. Harnessing inorganic nanoparticles to direct macrophage polarization for skeletal muscle regeneration. Nanomaterials (Basel). 2020;10(10).

31. de Haan JJ, Smeets MB, Pasterkamp G, Arslan F. Danger signals in the initiation of the inflammatory response after myocardial infarction. Mediat Inflamm. 2013;2013:206039.

32. Rennert RC, Sorkin M, Garg RK, Gurtner GC. Stem cell recruitment after injury: lessons for regenerative medicine. Regen Med. 2012;7(6):833-50.

33. Chen D, Xia Y, Zuo K, Wang Y, Zhang S, Kuang D, et al. Crosstalk between SDF-1/CXCR4 and SDF-1/CXCR7 in cardiac stem cell migration. Sci Rep. 2015;5:16813.

34. O'Rourke SA, Dunne A, Monaghan MG. The role of macrophages in the infarcted myocardium: orchestrators of ECM remodeling. Front Cardiovasc Med. 2019;6:101.

35. Steele AN, MacArthur JW, Woo YJ. Stem cell therapy: healing or hype? Why stem cell delivery doesn't work. Circ Res. 2017;120(12):1868-70.

36. Rawat S, Gupta S, Mohanty S. Mesenchymal stem cells modulate the immune system in developing therapeutic interventions. In: Tyagi RK, Bisen PS, editors. Immune response activation: IntechOpen; 2019.

37. Pan W, Zhu Y, Meng X, Zhang C, Yang Y, Bei Y. Immunomodulation by exosomes in myocardial infarction. $\mathrm{J}$ Cardiovasc Transl Res. 2018.

38. Zhao J, Li X, Hu J, Chen F, Qiao S, Sun X, et al. Mesenchymal stromal cell-derived exosomes attenuate myocardial ischaemiareperfusion injury through miR-182-regulated macrophage polarization. Cardiovasc Res. 2019;115(7):1205-16.

39. Teng X, Chen L, Chen W, Yang J, Yang Z, Shen Z. Mesenchymal stem cell-derived exosomes improve the microenvironment of infarcted myocardium contributing to angiogenesis and anti-inflammation. Cell Physiol Biochem. 2015;37(6):2415-24.

40. Gallet R, Dawkins J, Valle J, Simsolo E, de Couto G, Middleton R, et al. Exosomes secreted by cardiosphere-derived cells reduce scarring, attenuate adverse remodelling, and improve function in acute and chronic porcine myocardial infarction. Eur Heart J. 2017;38(3): 201-11. 
41. de Couto G, Gallet R, Cambier L, Jaghatspanyan E, Makkar N, Dawkins JF, et al. Exosomal microRNA transfer into macrophages mediates cellular postconditioning. Circulation. 2017;136(2):20014.

42. Bui QT, Gertz ZM, Wilensky RL. Intracoronary delivery of bonemarrow-derived stem cells. Stem Cell Res Ther. 2010;1(4):29.

43. Brusatin G, Panciera T, Gandin A, Citron A, Piccolo S. Biomaterials and engineered microenvironments to control YAP/ TAZ-dependent cell behaviour. Nat Mater. 2018;17(12):1063-75. This review discusses the key role of YAP/TAZ, two mechanosensitive transcriptional regulators, in the rational design of material-based platforms.

44. Ciocci M, Mochi F, Carotenuto F, Di Giovanni E, Prosposito P, Francini R, et al. Scaffold-in-scaffold potential to induce growth and differentiation of cardiac progenitor cells. Stem Cells Dev. 2017;26(19):1438-47. This experimental study introduces an innovative 3D composite scaffold fabrication approach in which a stiffer woodpile structure is embedded into a softer hydrogel to modulate cardiac cell differentiation and orientation.

45. Chachques JC, Lila N, Soler-Botija C, Martinez-Ramos C, Valles A, Autret G, et al. Elastomeric cardiopatch scaffold for myocardial repair and ventricular support. Eur J Cardiothorac Surg. 2020;57(3): 545-55.

46. Guyette JP, Charest JM, Mills RW, Jank BJ, Moser PT, Gilpin SE, et al. Bioengineering human myocardium on native extracellular matrix. Circ Res. 2016;118(1):56-72.

47. Bejleri D, Streeter BW, Nachlas ALY, Brown ME, Gaetani R, Christman KL, et al. A bioprinted cardiac patch composed of cardiac-specific extracellular matrix and progenitor cells for heart repair. Adv Healthc Mater. 2018;7(23):e1800672.

48. Bejleri D, Davis ME. Decellularized extracellular matrix materials for cardiac repair and regeneration. Adv Healthc Mater. 2019;8(5): e1801217.

49. Gazia C, Tamburrini R, Asthana A, Chaimov D, Muir SM, Marino DI, et al. Tissue engineering potential of ECM-based hydrogels obtained from human tissues. Ann Biomed Eng. 2019;24(5):604 12.

50. Edgar L, Altamimi A, Garcia Sanchez M, Tamburrinia R, Asthana A, Gazia C, et al. Utility of extracellular matrix powders in tissue engineering. Organogenesis. 2018;14(4):172-86.

51. Baiguera S, Del Gaudio C, Di Nardo P, Manzari V, Carotenuto F, Teodori L. 3D printing decellularized extracellular matrix to design biomimetic scaffolds for skeletal muscle tissue engineering. Biomed Res Int. 2020;2020:2689701.

52. Politi S, Carotenuto F, Rinaldi A, Di Nardo P, Manzari V, Albertini MC, et al. Smart ECM-based electrospun biomaterials for skeletal muscle regeneration. Nanomaterials (Basel). 2020;10(9).

53. Doostmohammadi M, Forootanfar $\mathrm{H}$, Ramakrishna S. Regenerative medicine and drug delivery: progress via electrospun biomaterials. Mater Sci Eng C Mater Biol Appl. 2020;109:110521.

54. Solazzo M, O'Brien FJ, Nicolosi V, Monaghan MG. The rationale and emergence of electroconductive biomaterial scaffolds in cardiac tissue engineering. APL Bioeng. 2019;3(4):041501.

55. Kowalski PS, Bhattacharya C, Afewerki S, Langer R. Smart biomaterials: recent advances and future directions. ACS Biomater Sci Eng. 2018;4(11):3809-17.
56. Gardin C, Ferroni L, Latremouille C, Chachques JC, Mitrecic D, Zavan B. Recent applications of three dimensional printing in cardiovascular medicine. Cells. 2020;9(3).

57. O'Brien CM, Holmes B, Faucett S, Zhang LG. Three-dimensional printing of nanomaterial scaffolds for complex tissue regeneration. Tissue Eng Part B Rev. 2015;21(1):103-14.

58. Shin YJ, Shafranek RT, Tsui JH, Walcott J, Nelson A, Kim DH. 3D bioprinting of mechanically tuned bioinks derived from cardiac decellularized extracellular matrix. Acta Biomater. 2021;119:7588.

59. Duan B. State-of-the-art review of 3D bioprinting for cardiovascular tissue engineering. Ann Biomed Eng. 2017;45(1):195-209.

60. Wilson WC Jr, Boland T. Cell and organ printing 1: protein and 752 cell printers. Anat Rec A Discov Mol Cell Evol Biol. 2003;272(2): $753 ; 491-6$. Historically important study in which the authors describe the development of the first inkjet bioprinter by modifying an HP standard inkjet printer.

61. $\mathrm{Gu} \mathrm{Z,} \mathrm{Fu} \mathrm{J,} \mathrm{Lin} \mathrm{H,} \mathrm{He} \mathrm{Y.} \mathrm{Development} \mathrm{of} \mathrm{3D} \mathrm{bioprinting:} \mathrm{from}$ printing methods to biomedical applications. Asian J Pharm Sci. 2020;15(5):529-57.

62. Zhang YS, Oklu R, Dokmeci MR, Khademhosseini A. Threedimensional bioprinting strategies for tissue engineering. Cold Spring Harb Perspect Med. 2018;8(2).

63.• Hinton TJ, Jallerat Q, Palchesko RN, Park JH, Grodzicki MS, Shue HJ, et al. Three-dimensional printing of complex biological structures by freeform reversible embedding of suspended hydrogels. Sci Adv. 2015;1(9):e1500758. This key paper shows the development of the FRESH technique enabling 3D bioprinting of soft biomaterials.

64. Maiullari F, Costantini M, Milan M, Pace V, Chirivi M, Maiullari S, et al. A multi-cellular 3D bioprinting approach for vascularized heart tissue engineering based on HUVECs and iPSC-derived cardiomyocytes. Sci Rep. 2018;8(1):13532.

65.• Noor N, Shapira A, Edri R, Gal I, Wertheim L, Dvir T. 3D printing of personalized thick and perfusable cardiac patches and hearts. Adv Sci (Weinh). 2019;6(11):1900344 The authors provide for the first time the proof of concept of printing patient-specific 3D cardiac constructs and cellularized human hearts using human tissue as bio-ink.

66.• Lee A, Hudson AR, Shiwarski DJ, Tashman JW, Hinton TJ, Yerneni S, et al. 3D bioprinting of collagen to rebuild components of the human heart. Science. 2019;365(6452):482-7. The authors refining the FRESH technique, demonstrate 3D printing of components of the human heart spanning capillary to full-organ scale.

67.• Mirdamadi E, Tashman JW, Shiwarski DJ, Palchesko RN, Feinberg AW. FRESH 3D bioprinting a full-size model of the human heart. ACS Biomater Sci Eng. 2020;6(11):6453-9. Feinberg and his team create the first full-size 3D bioprinted human heart model refining their FRESH technique.

Publisher's Note Springer Nature remains neutral with regard to jurisdictional claims in published maps and institutional affiliations. 